UCRL-JC-130763

Preprint

\title{
Edge Plasma Modeling of Limiter Surfaces in a Tokamak Divertor Configuration
}

\author{
M.E. Rensink, T.D. Rognlien
}

This paper was prepared for submittal to

13th International Conference on Plasma Surface Interactions

May 18-22, 1998

San Diego, CA

May 11, 1998

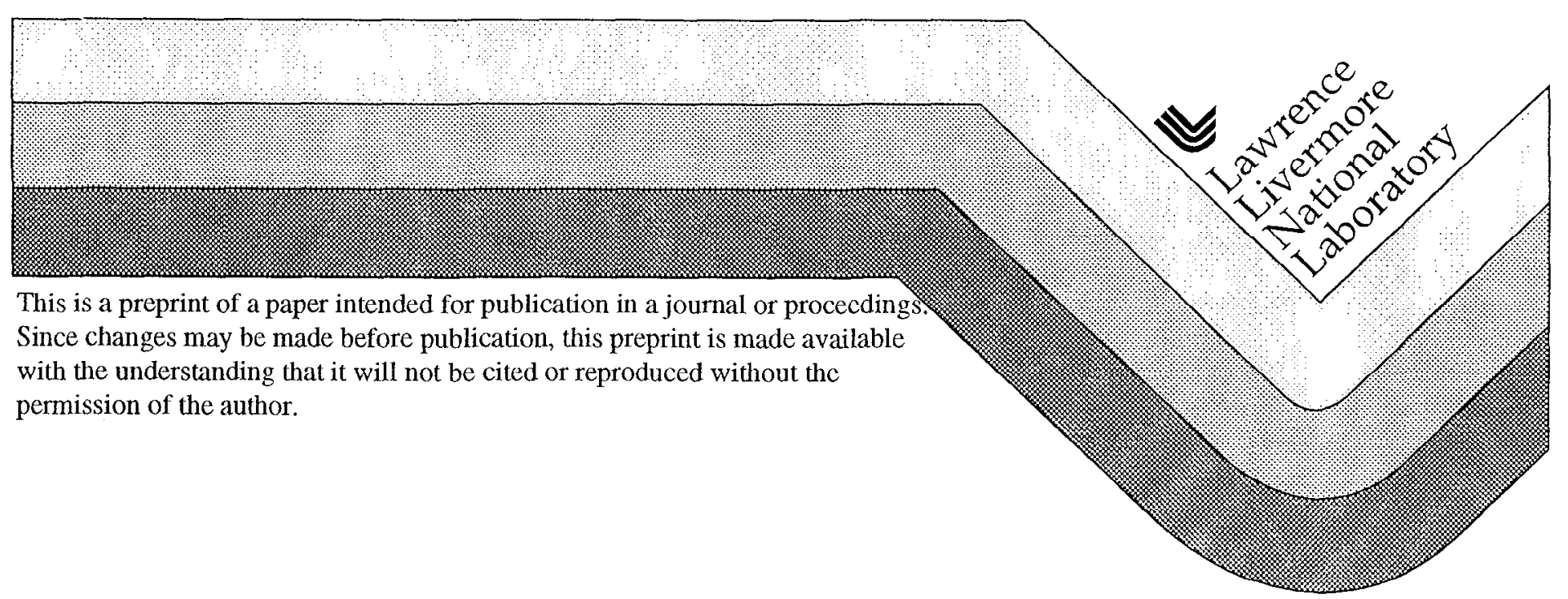




\section{DISCLAIMER}

This document was prepared as an account of work sponsored by an agency of the United States Government Neither the United States Government nor the University of California nor any of their employees, makes any warranty, express or implied, or assumes any legal liability or responsibility for the accuracy, completeness, or usefulness of any information, apparatus, product, or process disclosed, or represents that its use would not infringe puivately owned rights Reference herein to any specific commercial product, process, or service by trade name, trademark, manufacturer, or otherwise, does not necessarily constitute or imply its endorsement, recommendation, or favoring by the United States Government or the University of California. The views and opinions of authors expressed herein do not necessarily state or reflect those of the United States Government or the University of California, and shall not be used for advertising or product endorsement purposes 


\title{
Edge Plasma Modeling of Limiter Surfaces in a Tokamak Divertor Configuration
}

\author{
M. E. Rensink and T. D. Rognlien \\ Lawrence Livermore National Laboratory, Livermore, CA 94550, USA
}

\section{Introduction}

During the startup phase of a tokamak the plasma configuration may evolve from a limiter to a divertor configuration. Some of the particle and heat flux from the core will be deposited on material surfaces near the separatrix instead of the divertor plates. Examples of such surfaces include the center-post in most tokamaks, baffles near the x-point that create closed divertors, and outboard limiter surfaces. Two-dimensional edge plasma models for tokamak divertor configurations typically give detailed information about the particle and heat fluxes on the divertor plates, but yield little or no information about fluxes on these other localized surfaces near the core plasma. To realistically model the startup phase of a tokamak it is necessary to compute the plasma interaction with both limiter and divertor surfaces. The UEDGE code [1] has been modified to include these limiter surfaces. In this report we present simulation results for an idealized ITER [2] startup configuration with variations in the limiter penetration depth and surface shape.

\section{Model}

The UEDGE code is a fully implicit 2-D edge plasma transport code. It solves the classical Braginskii transport equations [3] for plasma density, parallei momentum and thermal ion and electron energy transport along the magnetic field, and assumes anomalous diffusive transport across the field. For the simulations in this report the anomalous particle diffusivity is $0.33 \mathrm{~m}^{2} / \mathrm{sec}$ and the thermal diffusivities are $0.5 \mathrm{~m}^{2} / \mathrm{sec}$ for both ions and electrons. The code uses a reduced Navier-Stokes model [4,5] for atomic neutrals with a single momentum equation for transport along field lines.

\subsection{Mesh Configuration}

The 2-D simulation includes the scrape-off-layer region outside the separatrix and a narrow region of the core plasma just inside the separatrix. One set of mesh surfaces coincides with magnetic flux surfaces; the second set of mesh surfaces is nearly orthogonal to the magnetic flux surfaces, except in the vicinity of the divertor plates and limiter where the mesh is distorted to conform to the shape of the recycling surface. Along each magnetic flux surface there are 112 cells and radially across magnetic flux surfaces there are 16 cells. Near the limiter and divertor surfaces the poloidal cell size is smaller (less than $1 \mathrm{~cm}$ ) in order to resolve the strong plasma and neutral fluid gradients associated with strong recycling. Radially, the cell size is smallest near the separatrix where strong radial gradients are expected The divertor plates in these simulations are orthogonal to magnetic flux surfaces. We use an idealized toroidally symmetric wedge-shaped limiter as shown in Figures 1 and 2 . The wedge angle and 
the penetration of the limiter tip relative to the separatrix are variable.. When the wedge angle is zero, the limiter is a toroidally symmetric infinitely-thin plate (a horizontal line when viewed in the poloidal plane) inserted edge-wise into the plasma. While this wedge-shaped limiter is not realistic, the SOL plasma parameters (density and temperatures at the LCFS and radial decay lengths) are relatively insensitive to the wedge angle.

\subsection{Boundary Conditions}

We assume standard recycling boundary conditions at the plasma-limiter interface, i.e., the neutral atom flux is $\Gamma_{\text {neutral }}=-R_{p} \cdot \Gamma_{\text {ion }}$ where $\Gamma_{\text {ion }}$ is the incident ion flux and $R_{p}$ is the particle recycling coefficient; the ion parallel flow velocity at the surface is sonic; and the ion energy flux is $\mathrm{Q}_{\text {ion }}=\delta_{\text {ion }} \cdot \mathrm{T}_{\text {ion }} \cdot \Gamma_{\text {ion }}$ where $\delta_{\text {ion }}$ is the sheath energy transmission coefficient for ions. A similar condition is used for electrons. The simulations in this report use a limiter particle recycling coefficient of 0.8 and ion(electron) energy transmission coefficients are $3.5(5.0)$. At the innermost core boundary, $0.6 \mathrm{~cm}$ inside the separatrix, the ion density $\mathrm{n}_{\text {core }}=$ $5 \times 10^{19} \mathrm{~m}^{-3}$ is fixed. The total radial power flow across the core boundary is $\mathrm{P}_{\text {core }}=150 \mathrm{MW}$ for modeling the ITER startup, equally divided between electrons and ions. At the divertor plates we assume 100 percent recycling, a sonic flow condition for ions and sheath energy transmission coefficients 3.5(5.0) for ions(electrons).

\section{Results}

The results presented here are steady state plasma simulations. The mesh is based on the ITER equilibrium magnetic configuration in which the flux surfaces outside the separatrix all terminate at the divertor plates. To simulate a startup configuration, we introduce a shaped limiter surface near the outboard midplane that intersects the flux surfaces and absorbs heat and particles that would have gone to the divertor plates. We vary the limiter surface shape so as to approach a 'conformal' shape that is nearly tangent to flux surfaces in the poloidal plane, as in the ITER reference design.

The first set of simulations examines the redistribution of the power between divertor and limiter surfaces as a thin limiter plate is inserted at the outboard midplane. The results in Figure 3 show that when the limiter tip is about $1 \mathrm{~cm}$ outside the separatrix flux surface the power is split equally between the limiter and divertor plates. If the limiter is inserted $0.5 \mathrm{~cm}$ beyond the separatrix, then essentially all of the power is deposited on the limiter. Besides the power re-distribution the limiter produces significant changes in the density profile of the SOL. Since the limiter particle recycling coefficient $R_{p}=0.8$ and there is a large ion particle flux onto the limiter, there is a large net particle removal rate by the limiter. For example, with the limiter tip at the separatrix flux surface the total ion current to the limiter is $150 \mathrm{kA}$ and the total particle removal rate is $30 \mathrm{kA}$. In our simulations these particles are supplied by a net current across the innermost core boundary. This radial particle current is associated with radial gradients in the SOL density profile due to limiter pumping. Figure 4 shows the steepening of the radial density profile in the SOL as the limiter is inserted to the separatrix.

A second set of simulations examines the effect of limiter shape on the heat flux profiles at the limiter surface. The results in Figure 5 show the heat flux profiles for several wedge shapes with the limiter tip exactly at the the separatrix. When the limiter surface is normal 10 llux surfaces, the maximum heat flux on the limiter is about $90 \mathrm{MW} / \mathrm{m}^{2}$. As expected, the maximum heat flux decreases to less than $30 \mathrm{MW} / \mathrm{m}^{2}$ as the surface is tilted to be more nearly parallel to magnetic flux surfaces. Simulations with larger wedge angles present numerical 
difficulties because the mesh beomces very distorted near the limiter surface. In the limit where the wedge angle is $180^{\circ}$ the limiter surface would represent a flat plate tangent to the magnetic flux surface, and we expect the heat flux at the tangent point to be very small; the heat flux would probably be a maximum at some point on the limiter that is further out in the SOL. In Fig. 5 the decrease in heat flux with increasing wedge angle is primarily due to the increase in the wetted area of the limiter. Changes also occur in the densily profile along flux surfaces in the vicinity of the limiter. Recycling near the limiter tip produces a localized ionization source and a peak in the plasma density profile as shown in Fig. 6. The limiter tip is at the separatrix, so the density peak occurs inside the separatrix and radial diffusion produces a localized influx of particles into the core region. However, the integrated particle flux across the core boundary is radially outward due to the net pumping by the limiter surface. In the absence of the limitcr, there is no net particle removal and the SOL density is nearly uniform in the radial and poloidal directions. The total energy and particles deposited on the limiter are not significantly affected by the limiter surface shape. With the limiter tip at the separatrix, the peak plasma temperature at the limiter surface is $170 \mathrm{eV}$ and the total ion particle flux on the limiter is about $150 \mathrm{kA}$, so impurity generation due to sputtering from the limiter could be a problem.

\section{Summary}

We have developed a capability for 2-D modeling of edge plasmas with localized limiter surfaces penetrating the SOL. The total power deposited on limiter and divertor surfaces is strongly dependent on the maximum penetration of the limiter, but is only weakly affected by changes in limiter shape. The peak heat flux on the limiter is reduced by tilting the limiter surface relative to the magnetic flux surfaces, but the total heat load on the limiter is nearly independent of the tilt for fixed limiter tip penetration. Particle pumping by the limiter induces strong radial gradients in the SOL plasma density and large ion currents from the core to the SOL.

\section{Acknowledgments}

This work was performed under the auspices of the US Department of Energy by the Lawrence Livermore National Laboratory under contract no. W-7405-ENG-48.

\section{References}

1. Rognlien, T.D., et al., Contr. Plasma Phys. 34 (1994) 362.

2. Parker, R., el al., J. Nucl. Mater. 241-243 (1997) 1.

3. Braginskii, S.I., Transport Processes in a Plasma, Revicws of Plasma Physics, Vol I, Ed. M.A. Leontovich (Consultants Bureau, New York, 1965), p. 205.

4. Knoll, D., Phys. Plasmas 3 (1996) 293.

5. Wising, F., et al., Contr. Plasma Phys. 36 (1996) 136. 


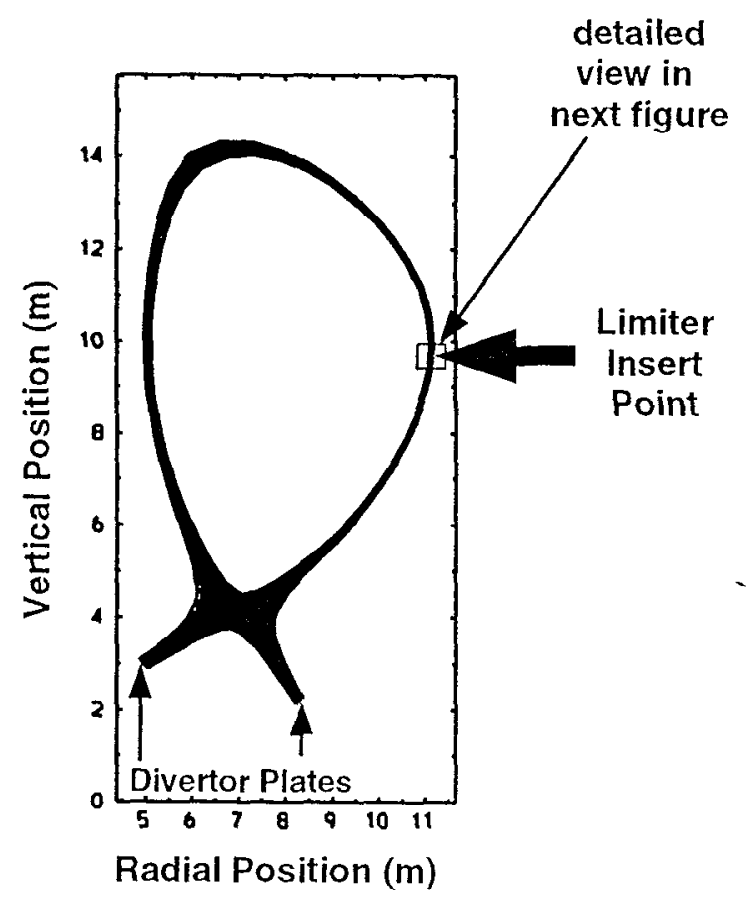

Fig 1. Global view of the simulation region and limiter insertion point 


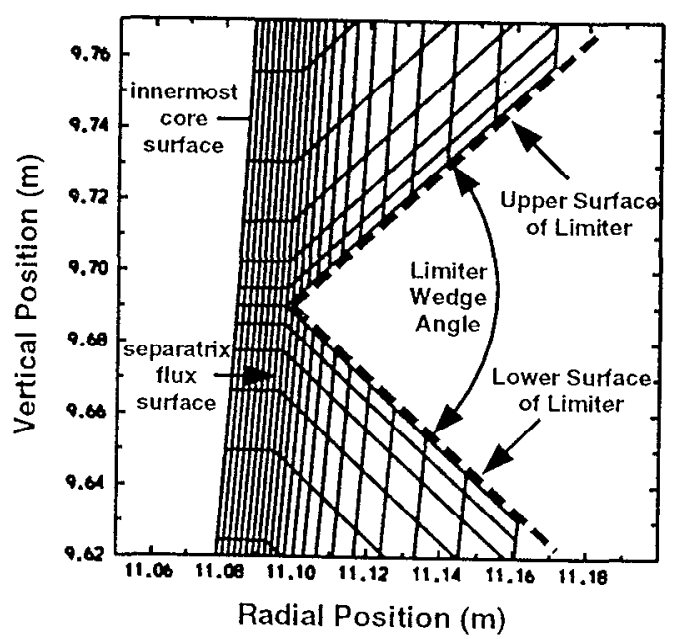

Fig. 2 Detailed view of the mesh and limiter configuration at the outboard midplane 


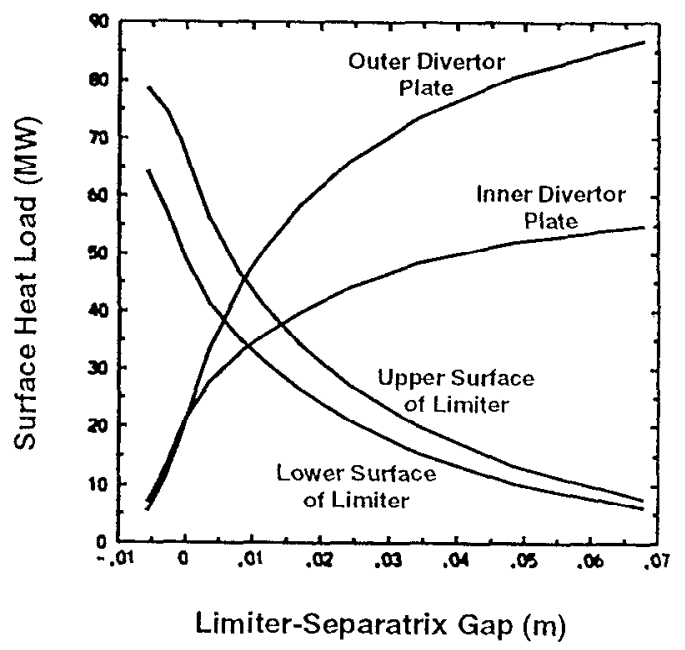

Fig 3 Variation of the total heat load on limiter and divertor surfaces as a function of the radial distance between the limiter tip and the separatrix flux suface The limiter wedge angle is zero 


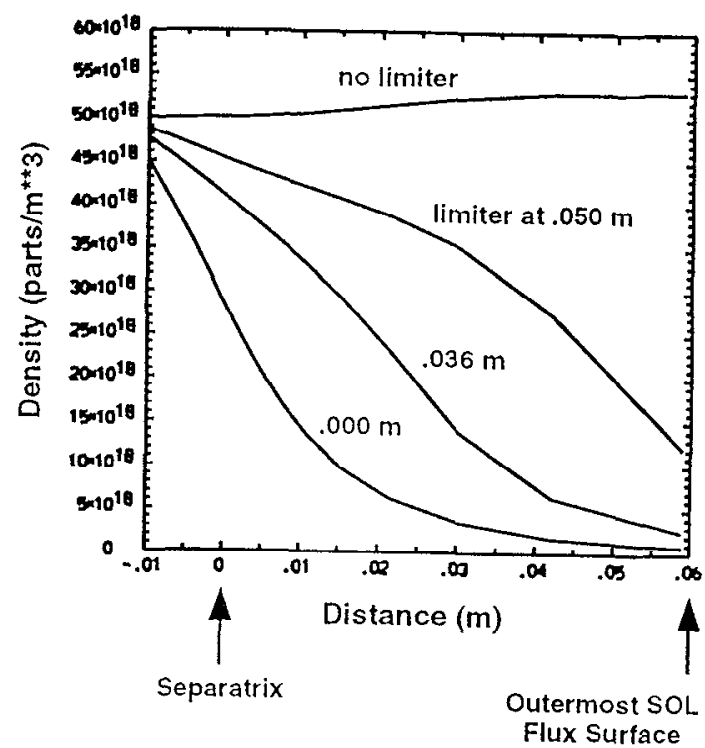

Fig 4 Radial profile of the plasma density for varying limiter penetration The profile is at a poloidal position half-way between the outboard midplane and the $x$-point 


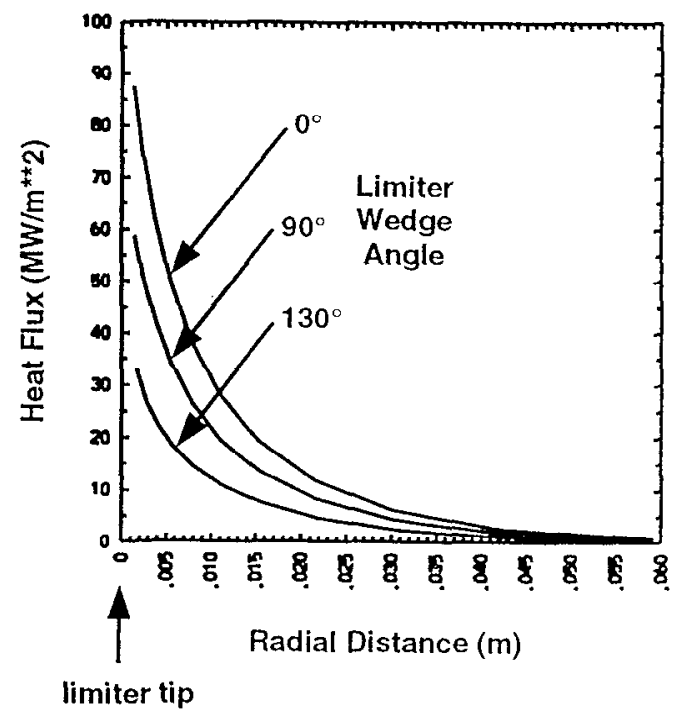

Fig. 5 Heat flux profile on the upper surface of the limiter as a function of radial distance from the separatrix for various wedge angles The limiter tip is at the separatrix flux surface 


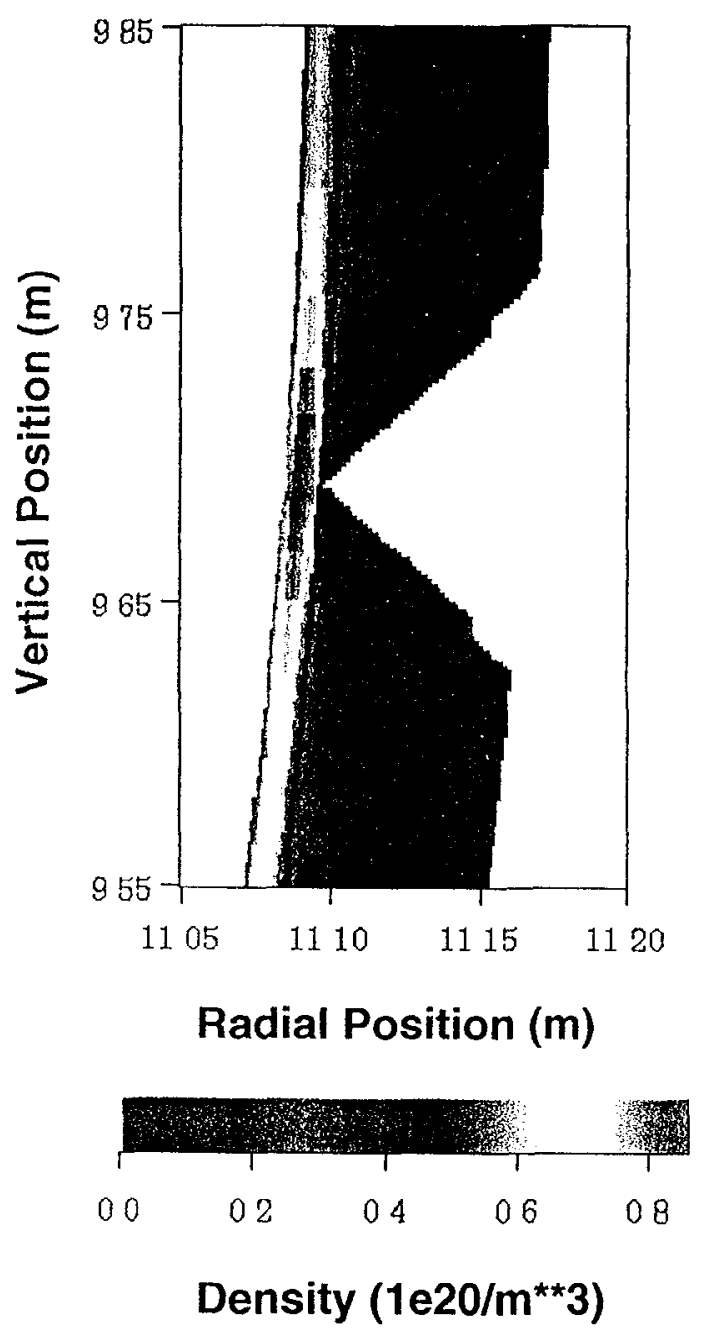

Fig. 6. Plasma density contours in the vicinity of the limiter. The limiter tip is at the separatrix flux surface and the limiter wedge angle is 90 degrees. 


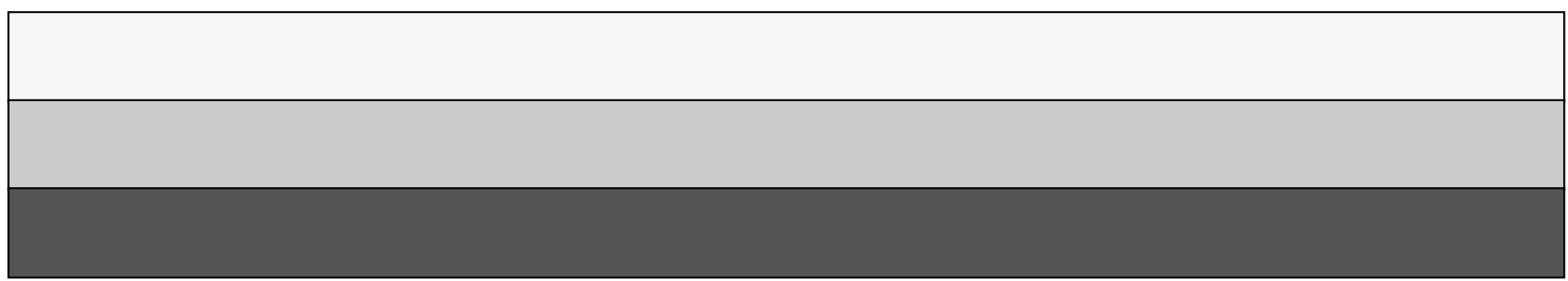

